Bull. Korean Math. Soc. 50 (2013), No. 2, pp. 611-626

http://dx.doi.org/10.4134/BKMS.2013.50.2.611

\title{
UNIFORM ASYMPTOTICS FOR THE FINITE-TIME RUIN \\ PROBABILITY IN A GENERAL RISK MODEL WITH \\ PAIRWISE QUASI-ASYMPTOTICALLY INDEPENDENT CLAIMS AND CONSTANT INTEREST FORCE
}

\author{
Qingwu GaO And Yang Yang
}

\begin{abstract}
In the paper we study the finite-time ruin probability in a general risk model with constant interest force, in which the claim sizes are pairwise quasi-asymptotically independent and arrive according to an arbitrary counting process, and the premium process is a general stochastic process. For the case that the claim-size distribution belongs to the consistent variation class, we obtain an asymptotic formula for the finite-time ruin probability, which holds uniformly for all time horizons varying in a relevant infinite interval. The obtained result also includes an asymptotic formula for the infinite-time ruin probability.
\end{abstract}

\section{Introduction}

In this paper, we consider a general risk model with the claim sizes, their arrival process and the premium process satisfying the following assumptions.

Assumption 1. The claim sizes $\left\{X_{i}, i \geq 1\right\}$ form a sequence of nonnegative, identically distributed, but not necessarily independent random variables (r.v.s) with common distribution $F$.

Assumption 2. The claim arrival process $\{N(t), t \geq 0\}$ is a general counting process, independent of $\left\{X_{i}, i \geq 1\right\}$ and such that $0<E N(t)<\infty$ for all $0<t<\infty$.

Assumption 3. The total amount of premiums accumulated up to time $t \geq 0$, denoted by $C(t)$, is a nonnegative and nondecreasing stochastic process with $C(0)=0$ and $C(t)<\infty$ almost surely for each $t>0$.

Received December 4, 2011; Revised July 10, 2012.

2010 Mathematics Subject Classification. 62P05, 62E20, 60F05.

Key words and phrases. uniform asymptotics, finite-time ruin probability, pairwise quasiasymptotic independence, consistent variation.

This paper is supported by National Natural Science Foundation of China (NO. 11001052), Natural Science Foundation of Jiangsu Province of China (NO. BK2010480) and Research Start-up Funding for PhD of Nanjing Audit University (NO. NSRC10022). 
Denote the arrival time of $i$-claim by $\tau_{i}, i \geq 1$. Let $r>0$ be the constant interest force, that is to say, a monetary unit of capital will become $e^{r t}$ after time $t$, and let $x \geq 0$ be the insurer's initial reserve. Hence, the total reserve up to time $t \geq 0$ of the insurance company, denoted by $U_{r}(t)$, satisfies

$$
U_{r}(t)=x e^{r t}+\int_{0-}^{t} e^{r(t-s)} C(d s)-\sum_{i=1}^{N(t)} X_{i} e^{r\left(t-\tau_{i}\right)}, \quad t \geq 0,
$$

where the summation over an empty set of index values is assumed to vanish.

As generally admitted, once the insurer's reserve becomes negative, then we say that the ruin occurs. The ruin probability within a finite time $t$ is defined by

$$
\psi_{r}(x, t)=P\left(U_{r}(s)<0 \text { for some } 0 \leq s \leq t\right),
$$

and the infinite-time ruin probability is

$$
\psi_{r}(x)=P\left(U_{r}(t)<0 \text { for some } t \geq 0\right) .
$$

Hereafter, all limit relationships are for $x \rightarrow \infty$ unless mentioned otherwise. For two positive functions $a(\cdot)$ and $b(\cdot)$, we write $a(x)=O(b(x))$ if $\limsup a(x) / b(x)=C<\infty$, write $a(x) \lesssim b(x)$ or $b(x) \gtrsim a(x)$ if $C \leq 1$, write $a(x) \sim b(x)$ if $a(x) \lesssim b(x)$ and $b(x) \lesssim a(x)$, write $a(x)=o(b(x))$ if $C=0$. For two positive bivariate functions $a(\cdot, \cdot)$ and $b(\cdot, \cdot)$, we say that relation $a(x, t) \sim b(x, t)$ holds uniformly for all $t \in \Delta \neq \emptyset$ if

$$
\lim _{x \rightarrow \infty} \sup _{t \in \Delta}\left|\frac{a(x, t)}{b(x, t)}-1\right|=0 .
$$

Now we introduce some important classes of heavy-tailed distributions, one of which is the subexponential class $\mathcal{S}$. Say that a distribution $V$ belongs to the class $\mathcal{S}$, denoted by $V \in \mathcal{S}$, if $\bar{V}(x)=1-V(x)>0$ for all $x>0$ and $\overline{V^{* 2}}(x) \sim 2 \bar{V}(x)$, where $V^{* 2}$ denotes the 2-fold convolution of $V$. Clearly, if $V \in \mathcal{S}$, then $V$ is long-tailed, denoted by $V \in \mathcal{L}$ and characterized by

$$
\bar{V}(x+y) \sim \bar{V}(x) \quad \text { for all } \quad y \neq 0 .
$$

Another important class is the dominated variation class $\mathcal{D}$. Say that a distribution $V$ belongs to the class $\mathcal{D}$, denoted by $V \in \mathcal{D}$, if $\bar{V}(x y)=O(\bar{V}(x))$ for all $y>0$. A slightly smaller class of $\mathcal{D}$ is the consistent variation class $\mathcal{C}$. Say that a distribution $V$ belongs to the class $\mathcal{C}$, denoted by $V \in \mathcal{C}$, if $\lim _{y \searrow 1} \liminf _{x \rightarrow \infty} \bar{V}(x y) / \bar{V}(x)=1$, or equivalently,

$$
\lim _{y \nearrow 1} \limsup _{x \rightarrow \infty} \bar{V}(x y) / \bar{V}(x)=1 .
$$

It is well-known that a proper inclusion relationships hold, namely

$$
\mathcal{C} \subset \mathcal{L} \cap \mathcal{D} \subset \mathcal{S} \subset \mathcal{L}
$$


For more details of heavy-tailed distributions and their applications to insurance and finance, we refer readers to Bingham et al. ([1]) and Embrechts et al. $([3])$.

As universally acknowledged, the finite-time ruin probability is more practical in ruin theory but much harder to research. For a risk model with constant interest force, when its claim sizes and inter-arrival times are two sequences of independent and identically distributed (i.i.d.) r.v.s, there are many works on asymptotics for the finite-time ruin probability (see Tang ([12, 13]), Hao and Tang ([4]), and references therein). Recently, Wang ([14]) showed that in a risk model, the claim sizes $\left\{X_{i}, i \geq 1\right\}$ are i.i.d. r.v.s with common distribution $F \in \mathcal{L} \cap \mathcal{D}$, and their arrival process $\{N(t), t \geq 0\}$ is a general counting process satisfying $E N(t)>0$ and $E(1+\epsilon)^{N(t)}<\infty$ for any fixed $t>0$ and some $\epsilon=\epsilon(t)>0$, and $\left\{X_{i}, i \geq 1\right\},\{N(t), t \geq 0\}$ and $\{C(t), t \geq 0\}$ are mutually independent, then for the fixed $t>0$,

$$
\psi_{r}(x, t) \sim \int_{0-}^{t} \bar{F}\left(x e^{r s}\right) d E N(s) .
$$

Note that the independence assumption on the claim sizes is unrealistic in most practical situations and it limits the usefulness of the obtained results to some extent. Hence, in this paper we will consider a risk model with the claim sizes following a certain dependence structure, which was introduced by Chen and Yuen ([2]) and Yi et al. ([17]).

Definition 1.1. Say that two nonnegative r.v.s $\xi_{1}$ and $\xi_{2}$ with distributions $V_{1}$ and $V_{2}$, respectively, are quasi-asymptotically independent if

$$
P\left(\xi_{1}>x, \xi_{2}>x\right)=o\left(\overline{V_{1}}(x)+\overline{V_{2}}(x)\right) .
$$

More generally, two real-valued r.v.s $\xi_{1}$ and $\xi_{2}$ are still said to be quasiasymptotically independent if relation (1.5) holds with $\left(\xi_{1}, \xi_{2}\right)$ on the lefthand side replaced by $\left(\xi_{1}^{+}, \xi_{2}^{+}\right),\left(\xi_{1}^{+}, \xi_{2}^{-}\right)$and $\left(\xi_{1}^{-}, \xi_{2}^{+}\right)$. Say that a sequence of r.v.s $\left\{\xi_{i}, i \geq 1\right\}$ are pairwise quasi-asymptotically independent if for all positive integers $i \neq j$, the two r.v.s $\xi_{i}$ and $\xi_{j}$ are quasi-asymptotically independent.

Remark 1.1. We remark that the pairwise quasi-asymptotic independence allows a wide range of dependence structures among r.v.s, for example, the Farlie-Gumbel-Morgenstern family of distributions (see Kotz et al. ([7])) can provide a simple mechanism to construct an $n$-dimensional joint distribution such that its corresponding $n$ r.v.s are pairwise quasi-asymptotically independent. Also the pairwise quasi-asymptotic independence structure is wider than some commonly used dependence structures, such as widely upper orthant dependence/widely lower orthant dependence (WUOD/WLOD) (see Wang et al. $([15]))$.

Recently, more and more attention has been paid to the finite-time ruin probability in some dependent risk models with the claim sizes and/or their 
inter-arrival times satisfying a certain dependence structure, for example, see Kong and Zong ([6]), Yang and Wang ([16]), Wang et al. ([15]), among others. In particular, Wang et al. ([15]) considered a nonstandard risk model with WUOD claim sizes and WLOD inter-arrival times, and assumed that $\left\{X_{i}, i \geq\right.$ $1\},\{N(t), t \geq 0\}$ and $\{C(t), t \geq 0\}$ are mutually independent, they proved that if $F \in \mathcal{L} \cap \mathcal{D}$, then relation (1.4) holds uniformly for all $t$ in a finite interval.

Motivated by the results cited above, in the paper we aim at establishing an asymptotic formula for the finite-time ruin probability of a general risk model with pairwise quasi-asymptotically independent claim sizes arriving according to an arbitrary counting process, with a feature that the asymptotic formula holds uniformly for all times in a relevant infinite interval. In our main results, we will discuss two cases, one is the case that the premium process $\{C(t), t \geq 0\}$ is independent of $\left\{X_{i}, i \geq 1\right\}$ and $\{N(t), t \geq 0\}$, another is that $\{C(t), t \geq 0\}$ is not necessarily independent of $\left\{X_{i}, i \geq 1\right\}$ or $\{N(t), t \geq 0\}$.

In the remaining part of this paper, we will present the main results in Section 2, and prove them in Section 4 after giving some lemmas in Section 3.

\section{Main results}

In the section, we will state our main results of the paper. First, we introduce some notation. For a distribution $V$ and $y>0$, we set

$$
J_{V}^{+}=-\lim _{y \rightarrow \infty} \log \bar{V}_{*}(y) / \log y \quad \text { with } \quad \bar{V}_{*}(y)=\liminf _{x \rightarrow \infty} \bar{V}(x y) / \bar{V}(x) .
$$

Define $\Lambda=\{t: E N(t)>0\}=\left\{t: P\left(\tau_{1} \leq t\right)>0\right\}$ for a general counting process $\{N(t), t \geq 0\}$, and write $\underline{t}=\inf \{t: E N(t)>0\}=\inf \left\{t: P\left(\tau_{1} \leq t\right)>\right.$ $0\}$. It is clear that

$$
\Lambda= \begin{cases}{[\underline{t}, \infty],} & \text { if } \quad P\left(\tau_{1}=\underline{t}\right)>0 \\ (\underline{t}, \infty], & \text { if } \quad P\left(\tau_{1}=\underline{t}\right)=0 .\end{cases}
$$

Furthermore, we assume that the total discounted amount of premiums is finite, that is,

$$
0 \leq \widetilde{C}=\int_{0-}^{\infty} e^{-r t} C(d t)<\infty \text { almost surely. }
$$

Theorem 2.1. Consider the general risk model introduced in Section 1, where the claim sizes $X_{i}, i \geq 1$, are pairwise quasi-asymptotically independent with common distribution $F \in \mathcal{C}$, and for any fixed $t>0$, there exists some $p>J_{F}^{+}$ such that $E(N(t))^{p+1}<\infty$. Then, for any fixed $t_{0} \in \Lambda \cap(0, \infty)$, the relation (1.4) holds uniformly for all $t \in\left[t_{0}, \infty\right]$, if one of the following conditions is true:

1. the premium process $\{C(t), t \geq 0\}$ is independent of $\left\{X_{i}, i \geq 1\right\}$ and $\{N(t), t \geq 0\}$

2. the total discounted amount of premiums $\widetilde{C}$ satisfies that

$$
P(\widetilde{C}>x)=o(\bar{F}(x)) .
$$


Apparently, the condition on the general counting process that $E(N(t))^{p+1}<$ $\infty$ for some $p>J_{F}^{+}$is weaker than that of Wang ([14]), namely $E(1+\epsilon)^{N(t)}<\infty$ for some $\epsilon>0$, for any fixed $t>0$. According to Theorem 2.1, we can immediately derive the corresponding result for the infinite-time ruin probability.

Corollary 2.1. Under the conditions of Theorem 2.1, it holds that

$$
\psi_{r}(x) \sim \int_{0-}^{\infty} \bar{F}\left(x e^{r t}\right) d E N(t) .
$$

Remark 2.2. For Theorem 2.1 and Corollary 2.1 above, condition 1 has been considered by Tang ([12]), Wang ([14]), Wang et al. ([15]), among others, while as mentioned by Tang ([12]), condition 2 allows for a more realistic case that the premium rate varies as a deterministic or stochastic function of the insurer's current reserve, which does not require the independence between the premium process and the claim process, and was also considered by Petersen ([9]), Michaud ([8]) and Jasiulewicz ([5]).

\section{Lemmas}

In order to prove Theorem 2.1, we need a series of lemmas, among which the first lemma is a combination of Proposition 2.2.1 of Bingham et al. ([1]) and Lemma 3.5 of Tang and Tsitsiashvili ([10]).

Lemma 3.1. If a distribution $V \in \mathcal{D}$, then

(1) for any $p>J_{V}^{+}$, there are positive constants $C$ and $D$ such that

$$
\frac{\bar{V}(y)}{\bar{V}(x)} \leq C(x / y)^{p} \quad \text { for all } x \geq y \geq D ;
$$

(2) for any $p>J_{V}^{+}$, it holds that

$$
x^{-p}=o(\bar{V}(x))
$$

The following second lemma will play an important role in proving the main results, and is also of its own value and in the spirit of Proposition 5.1 of Tang and Tsitsiashvili ([11]).

Lemma 3.2. If $\left\{\xi_{i}, 1 \leq i \leq n\right\}$ are $n$ pairwise quasi-asymptotically independent and real-valued r.v.s with distributions $V_{i} \in \mathcal{L} \cap \mathcal{D}, 1 \leq i \leq n$, respectively, then for any fixed $0<a \leq b<\infty$,

$$
P\left(\sum_{i=1}^{n} c_{i} \xi_{i}>x\right) \sim \sum_{i=1}^{n} P\left(c_{i} \xi_{i}>x\right)
$$

holds uniformly for all $\underline{c_{n}}=\left(c_{1}, c_{2}, \ldots, c_{n}\right) \in[a, b]^{n}$. 
Proof. It should be mentioned that the idea of this proof is mainly from that of Chen and Yuen ([2]). Relation (3.3) is trivial if $n=1$. Herewith we assume $n \geq 2$. On the one hand, for an arbitrarily fixed $v \in(1 / 2,1)$,

$$
P\left(\sum_{i=1}^{n} c_{i} \xi_{i}>x\right) \leq P\left(\bigcup_{i=1}^{n}\left(c_{i} \xi_{i}>v x\right)\right)+P\left(\sum_{i=1}^{n} c_{i} \xi_{i}>x, \bigcap_{i=1}^{n}\left(c_{i} \xi_{i} \leq v x\right)\right)
$$

$$
=I_{1}+I_{2} \text {. }
$$

For $I_{1}$, by $V_{i} \in \mathcal{C}, 1 \leq i \leq n$, and the arbitrariness of $v \in(1 / 2,1)$, we have

(3.5) $\lim _{v \nearrow 1} \limsup _{x \rightarrow \infty} \frac{I_{1}}{\sum_{i=1}^{n} P\left(c_{i} \xi_{i}>x\right)} \leq \lim _{v \nearrow 1} \limsup _{x \rightarrow \infty} \frac{\sum_{i=1}^{n} P\left(c_{i} \xi_{i}>v x\right)}{\sum_{i=1}^{n} P\left(c_{i} \xi_{i}>x\right)}=1$.

For $I_{2}$, it follows that

$$
\begin{aligned}
I_{2} & =P\left(\sum_{i=1}^{n} c_{i} \xi_{i}>x, \frac{x}{n}<\max _{1 \leq k \leq n} c_{k} \xi_{k} \leq v x\right) \\
& \leq \sum_{k=1}^{n} P\left(\sum_{i=1, i \neq k}^{n} c_{i} \xi_{i}>(1-v) x, c_{k} \xi_{k}>\frac{x}{n}\right) \\
& \leq \sum_{k=1}^{n} \sum_{i=1, i \neq k}^{n} P\left(c_{i} \xi_{i}>\frac{(1-v) x}{n-1}, c_{k} \xi_{k}>\frac{x}{n}\right) .
\end{aligned}
$$

Since $\frac{1}{n}>\frac{1-v}{n-1}$, we derive from (1.5), (3.6) and $V_{i} \in \mathcal{C} \subset \mathcal{D}, 1 \leq i \leq n$, that

$$
\begin{aligned}
& \lim _{x \rightarrow \infty} \frac{I_{2}}{\sum_{i=1}^{n} P\left(c_{i} \xi_{i}>x\right)} \\
\leq & \lim _{x \rightarrow \infty} \sum_{k=1}^{n} \sum_{i=1, i \neq k}^{n} \frac{P\left(c_{i} \xi_{i}>\frac{(1-v) x}{n-1}, c_{k} \xi_{k}>\frac{x}{n}\right)}{P\left(c_{i} \xi_{i}>x\right)+P\left(c_{k} \xi_{k}>x\right)} \\
\leq & \lim _{x \rightarrow \infty} \sum_{k=1}^{n} \sum_{i=1, i \neq k}^{n} \frac{P\left(\xi_{i}>\frac{(1-v) x}{b(n-1)}, \xi_{k}>\frac{(1-v) x}{b(n-1)}\right)}{\overline{V_{i}}\left(\frac{(1-v) x}{b(n-1)}\right)+\overline{V_{k}}\left(\frac{(1-v) x}{b(n-1)}\right)} \\
& \cdot \frac{\overline{V_{i}}\left(\frac{(1-v) x}{b(n-1)}\right)+\overline{V_{k}}\left(\frac{(1-v) x}{b(n-1)}\right)}{\overline{V_{i}}(x / a)+\overline{V_{k}}(x / a)} \\
= & 0 .
\end{aligned}
$$

Thus, substituting (3.5) and (3.7) into (3.4) yields that

$$
P\left(\sum_{i=1}^{n} c_{i} \xi_{i}>x\right) \lesssim \sum_{i=1}^{n} P\left(c_{i} \xi_{i}>x\right)
$$

holds uniformly for all $\underline{c_{n}} \in[a, b]^{n}$. 
On the other hand, for an arbitrarily fixed $w>1$,

$$
\begin{aligned}
& P\left(\sum_{i=1}^{n} c_{i} \xi_{i}>x\right) \\
\geq & P\left(\sum_{i=1}^{n} c_{i} \xi_{i}>x, \max _{1 \leq k \leq n} c_{k} \xi_{k}>w x\right) \\
\geq & \sum_{k=1}^{n} P\left(\sum_{i=1}^{n} c_{i} \xi_{i}>x, c_{k} \xi_{k}>w x\right)-\sum_{1 \leq i<j \leq n} P\left(c_{i} \xi_{i}>w x, c_{j} \xi_{j}>w x\right)
\end{aligned}
$$

$(3.9)=I_{3}-I_{4}$.

For $I_{3}$, it holds that

$$
\begin{aligned}
I_{3} & \geq \sum_{k=1}^{n} P\left(c_{k} \xi_{k}>w x, \sum_{i=1, i \neq k}^{n} c_{i} \xi_{i}>(1-w) x\right) \\
& \geq \sum_{k=1}^{n} P\left(c_{k} \xi_{k}>w x, c_{i} \xi_{i}>(1-w) x, 1 \leq i \leq n, i \neq k\right) \\
& \geq \sum_{k=1}^{n} P\left(c_{k} \xi_{k}>w x\right)-\sum_{k=1}^{n} \sum_{i=1, i \neq k}^{n} P\left(c_{k} \xi_{k}>w x, c_{i} \xi_{i}<(1-w) x\right) \\
& =\sum_{k=1}^{n} P\left(c_{k} \xi_{k}>w x\right)-I_{5} .
\end{aligned}
$$

By the pairwise quasi-asymptotic independence property and $V_{i} \in \mathcal{C} \subset \mathcal{D}$, $1 \leq i \leq n$, we obtain that

$$
\begin{aligned}
& \limsup _{x \rightarrow \infty} \frac{I_{5}}{\sum_{i=1}^{n} P\left(c_{i} \xi_{i}>x\right)} \\
\leq & \limsup _{x \rightarrow \infty} \sum_{k=1}^{n} \sum_{i=1, i \neq k}^{n} \frac{P\left(c_{k} \xi_{k}>w x, c_{i} \xi_{i}<(1-w) x\right)}{P\left(c_{k} \xi_{k}>x\right)+P\left(c_{i} \xi_{i}>x\right)} \\
\leq & \limsup _{x \rightarrow \infty} \sum_{k=1}^{n} \sum_{i=1, i \neq k}^{n} \frac{P\left(\xi_{k}>(w-1) x / b, \xi_{i}<(1-w) x / b\right)}{\overline{V_{k}}((w-1) x / b)+\overline{V_{i}}((w-1) x / b)} \\
& \cdot \frac{\overline{V_{k}}((w-1) x / b)+\overline{V_{i}}((w-1) x / b)}{\overline{V_{k}}(x / a)+\overline{V_{i}}(x / a)} \\
= & 0 .
\end{aligned}
$$

This, along with (3.10), leads to

$$
\liminf _{x \rightarrow \infty} \frac{I_{3}}{\sum_{i=1}^{n} P\left(c_{i} \xi_{i}>x\right)} \geq \liminf _{x \rightarrow \infty} \frac{\sum_{k=1}^{n} P\left(c_{k} \xi_{k}>w x\right)}{\sum_{k=1}^{n} P\left(c_{k} \xi_{k}>x\right)} .
$$


For $I_{4}$, by $(1.5)$ and $V_{i} \in \mathcal{C} \subset \mathcal{D}, 1 \leq i \leq n$, we see that

$$
\begin{aligned}
& \limsup _{x \rightarrow \infty} \frac{I_{4}}{\sum_{i=1}^{n} P\left(c_{i} \xi_{i}>x\right)} \\
\leq & \limsup _{x \rightarrow \infty} \sum_{1 \leq i<j \leq n} \frac{P\left(c_{i} \xi_{i}>x, c_{j} \xi_{j}>x\right)}{P\left(c_{i} \xi_{i}>x\right)+P\left(c_{j} \xi_{j}>x\right)} \\
\leq & \limsup _{x \rightarrow \infty} \sum_{1 \leq i<j \leq n} \frac{P\left(\xi_{i}>x / b, \xi_{j}>x / b\right)}{\overline{V_{i}}(x / b)+\overline{V_{j}}(x / b)} \cdot \frac{\overline{V_{i}}(x / b)+\overline{V_{j}}(x / b)}{\overline{V_{i}}(x / a)+\overline{V_{j}}(x / a)} \\
= & 0 .
\end{aligned}
$$

From (3.9) to (3.12), and by $V_{i} \in \mathcal{C}, 1 \leq i \leq n$, and the arbitrariness of $w>1$, it follows that

$$
\lim _{w \searrow 1} \liminf _{x \rightarrow \infty} \frac{P\left(\sum_{i=1}^{n} c_{i} \xi_{i}>x\right)}{\sum_{i=1}^{n} P\left(c_{i} \xi_{i}>x\right)} \geq \lim _{w \searrow 1} \liminf _{x \rightarrow \infty} \frac{\sum_{i=1}^{n} P\left(c_{i} \xi_{i}>w x\right)}{\sum_{i=1}^{n} P\left(c_{i} \xi_{i}>x\right)}=1,
$$

which implies that

$$
P\left(\sum_{i=1}^{n} c_{i} \xi_{i}>x\right) \gtrsim \sum_{i=1}^{n} P\left(c_{i} \xi_{i}>x\right)
$$

holds uniformly for all $\underline{c_{n}} \in[a, b]^{n}$.

Consequently, we will complete the proof of this lemma by combining (3.8) and (3.13).

The following third lemma is due to Lemma 3.5 of Wang ([14]).

Lemma 3.3. For the general risk model introduced in Section 1, we have

$$
\sum_{i=1}^{\infty} P\left(X_{i} e^{-r \tau_{i}} \mathbf{1}_{\left\{\tau_{i} \leq t\right\}}>x\right)=\int_{0-}^{t} \bar{F}\left(x e^{r s}\right) d E N(s),
$$

where $\mathbf{1}_{A}$ is the indicator function of an event $A$.

Let $D_{r}(t)=\sum_{i=1}^{\infty} X_{i} e^{-r \tau_{i}} \mathbf{1}_{\left\{\tau_{i} \leq t\right\}}$ denote the discounted aggregated claims of the risk model in Section 1. Finally, we will discuss the uniform asymptotics of the probability $P\left(D_{r}(t)>x\right)$ which plays a key role for the proof of Theorem 2.1 .

Lemma 3.4. In the general risk model introduced in Section 1, if the claim sizes $X_{i}, i \geq 1$, are pairwise quasi-asymptotically independent with common distribution $F \in \mathcal{C}$, and their arrival process $\{N(t), t \geq 0\}$ satisfies $E(N(t))^{p+1}<$ $\infty$ for some $p>J_{F}^{+}$and any fixed $t>0$, then for any fixed $t_{0} \in \Lambda \cap(0, \infty)$, it holds uniformly for all $t \in\left[t_{0}, \infty\right]$ that

$$
P\left(D_{r}(t)>x\right) \sim \int_{0-}^{t} \bar{F}\left(x e^{r s}\right) d E N(s) .
$$


Proof. The idea is inspired by the proof of Theorem 1.1 of Tang ([13]). First, we prove that relation (3.14) holds uniformly for all $t \in\left[t_{0}, T\right]$, for an arbitrarily fixed $T \in\left(t_{0}, \infty\right)$. Note that, for all $x>0$,

$$
\begin{aligned}
P\left(D_{r}(t)>x\right) & =\left(\sum_{n=1}^{m}+\sum_{n=m+1}^{\infty}\right) P\left(\sum_{i=1}^{n} X_{i} e^{-r \tau_{i}}>x, N(t)=n\right) \\
& =K_{1}+K_{2},
\end{aligned}
$$

where $m \geq 1$ is a temporarily fixed integer. Let $D>0$ be the one appearing in (3.1) and such that $m+1 \leq x / D$. Now consider $K_{2}$, which is divided into two parts as

$$
\begin{aligned}
K_{2} & \leq\left(\sum_{m<n \leq x / D}+\sum_{x / D<n<\infty}\right) P\left(\sum_{i=1}^{n} X_{i}>x\right) P(N(t)=n), \\
& =K_{21}+K_{22},
\end{aligned}
$$

where the method of the division comes from Chen and Yuen ([2]). For $K_{21}$, by $F \in \mathcal{C} \subset \mathcal{D}$ and Lemma 3.1(1), we have

$$
\begin{aligned}
K_{21} & \leq \sum_{m<n \leq x / D} n \bar{F}(x / n) P(N(t)=n) \\
& \leq C \bar{F}(x) \sum_{m<n \leq x / D} n^{p+1} P(N(t)=n) \\
& \leq C \bar{F}(x) E(N(t))^{p+1} \mathbf{1}_{\{N(t)>m\}},
\end{aligned}
$$

where $p>J_{F}^{+}$and $C>0$ are the two constants as that in (3.1). For $K_{22}$, it follows from Markov's inequality that

$$
K_{22} \leq P(N(t)>x / D) \leq(x / D)^{-p-1} E(N(t))^{p+1} \mathbf{1}_{\{N(t)>x / D\}} .
$$

Substituting (3.17) and (3.18) into (3.16) and using Lemma 3.1(2) implies that, uniformly for all $t \in\left[t_{0}, T\right]$,

$$
K_{2} \lesssim C \bar{F}(x) E(N(t))^{p+1} \mathbf{1}_{\{N(t)>m\}} .
$$

Again by Lemma 3.1(1), we know that for all large $x$,

$$
\bar{F}(x) \leq C e^{r p t_{0}} \bar{F}\left(x e^{r t_{0}}\right) \leq C_{0} \int_{0-}^{t_{0}} \bar{F}\left(x e^{r s}\right) d E N(s),
$$

where $C_{0}=C e^{r p t_{0}} / E N\left(t_{0}\right)$. Hence, from (3.19), (3.20) and the condition on $\{N(t), t \geq 0\}$, we obtain that

$$
\begin{aligned}
& \lim _{m \rightarrow \infty} \sup _{t \in\left[t_{0}, T\right]} \frac{K_{2}}{\int_{0-}^{t} \bar{F}\left(x e^{r s}\right) d E N(s)} \\
\leq & \lim _{m \rightarrow \infty} \sup _{t \in\left[t_{0}, T\right]} \frac{C C_{0} E(N(t))^{p+1} \mathbf{1}_{\{N(t)>m\}} \int_{0-}^{t_{0}} \bar{F}\left(x e^{r s}\right) d E N(s)}{\int_{0-}^{t_{0}} \bar{F}\left(x e^{r s}\right) d E N(s)}
\end{aligned}
$$




$$
=0 .
$$

Next, consider $K_{1}$, let $G\left(t_{1}, \ldots, t_{n+1}\right)$ be the joint distribution of random vector $\left(\tau_{1}, \ldots, \tau_{n+1}\right)$, where $1 \leq n \leq m$. Then, by Lemma 3.2 and the independence between $\left\{X_{i}, i \geq 1\right\}$ and $\{N(t), t \geq 0\}$, it holds uniformly for all $t \in\left[t_{0}, T\right]$ and $1 \leq n \leq m$ that

$$
\begin{aligned}
& P\left(\sum_{i=1}^{n} X_{i} e^{-r \tau_{i}}>x, N(t)=n\right) \\
= & \int_{\left\{0 \leq t_{1} \leq \ldots \leq t_{n} \leq t, t_{n+1}>t\right\}} P\left(\sum_{i=1}^{n} X_{i} e^{-r t_{i}}>x\right) d G\left(t_{1}, \ldots, t_{n+1}\right) \\
\sim & \sum_{i=1}^{n} \int_{\left\{0 \leq t_{1} \leq \ldots \leq t_{n} \leq t, t_{n+1}>t\right\}} P\left(X_{i} e^{-r t_{i}}>x\right) d G\left(t_{1}, \ldots, t_{n+1}\right) \\
= & \sum_{i=1}^{n} P\left(X_{i} e^{-r \tau_{i}}>x, N(t)=n\right) .
\end{aligned}
$$

Thus, we have that, uniformly for all $t \in\left[t_{0}, T\right]$,

$$
\begin{aligned}
K_{1} & \sim \sum_{n=1}^{m} \sum_{i=1}^{n} P\left(X_{i} e^{-r \tau_{i}}>x, N(t)=n\right) \\
& =\left(\sum_{n=1}^{\infty}-\sum_{n=m+1}^{\infty}\right) \sum_{i=1}^{n} P\left(X_{i} e^{-r \tau_{i}}>x, N(t)=n\right) \\
& =K_{11}-K_{12} .
\end{aligned}
$$

Clearly, by Lemma 3.3, we derive that for all $t \in\left[t_{0}, T\right]$,

$$
\begin{aligned}
K_{11} & =\sum_{i=1}^{\infty} \sum_{n=i}^{\infty} P\left(X_{i} e^{-r \tau_{i}}>x, N(t)=n\right) \\
& =\sum_{i=1}^{\infty} P\left(X_{i} e^{-r \tau_{i}} \mathbf{1}_{\left\{\tau_{i} \leq t\right\}}>x\right) \\
& =\int_{0-}^{t} \bar{F}\left(x e^{r s}\right) d E N(s) .
\end{aligned}
$$

For $K_{12}$, it follows that

$$
K_{12} \leq \bar{F}(x) \sum_{n=m+1}^{\infty} n P(N(t)=n)=\bar{F}(x) E N(t) \mathbf{1}_{\{N(t)>m\}} .
$$

Similarly as in the derivation of (3.21), we still obtain that

$$
\lim _{m \rightarrow \infty} \sup _{t \in\left[t_{0}, T\right]} \frac{K_{12}}{\int_{0-}^{t} \bar{F}\left(x e^{r s}\right) d E N(s)}=0 .
$$


Therefore, combining (3.15), (3.21)-(3.24) can prove that relation (3.14) holds uniformly for all $t \in\left[t_{0}, T\right]$.

Subsequently, we extend the uniformity of relation (3.14) to an infinite interval $\left[t_{0}, \infty\right]$. By Lemma 3.1(1) again, it holds for all $t \in \Lambda$ and all large $x$ that

$$
\frac{\int_{t}^{\infty} \bar{F}\left(x e^{r s}\right) d E N(s)}{\int_{0-}^{t} \bar{F}\left(x e^{r s}\right) d E N(s)}=\frac{\int_{t}^{\infty} \bar{F}\left(x e^{r s}\right) / \bar{F}\left(x e^{r t}\right) d E N(s)}{\int_{0-}^{t} \bar{F}\left(x e^{r s}\right) / \bar{F}\left(x e^{r t}\right) d E N(s)} \leq C^{2} \frac{\int_{t}^{\infty} e^{-p r s} d E N(s)}{\int_{0-}^{t} e^{-p r s} d E N(s)}
$$

which gives

$$
\lim _{t \rightarrow \infty} \limsup _{x \rightarrow \infty} \frac{\int_{t}^{\infty} \bar{F}\left(x e^{r s}\right) d E N(s)}{\int_{0-}^{t} \bar{F}\left(x e^{r s}\right) d E N(s)}=0 .
$$

Thus, for arbitrarily fixed $\varepsilon>0$, there exists some large $T_{0} \in \Lambda$ such that for all large $x$,

$$
\int_{T_{0}}^{\infty} \bar{F}\left(x e^{r s}\right) d E N(s) \leq \varepsilon \int_{0-}^{T_{0}} \bar{F}\left(x e^{r s}\right) d E N(s) .
$$

Applying Remark 2 of Yi et al. ([17]), we find that if $F \in \mathcal{C}$, then

$$
P\left(D_{r}(\infty)>x\right) \sim \sum_{i=1}^{\infty} P\left(X_{i} e^{-r \tau_{i}}>x\right)=\int_{0-}^{\infty} \bar{F}\left(x e^{r s}\right) d E N(s),
$$

which means that relation (3.14) holds for $t=\infty$.

On the one hand, by (3.25) and (3.26), it holds uniformly for all $t \in\left(T_{0}, \infty\right]$ that

$$
\begin{aligned}
P\left(D_{r}(t)>x\right) & \leq P\left(D_{r}(\infty)>x\right) \sim \int_{0-}^{\infty} \bar{F}\left(x e^{r s}\right) d E N(s) \\
& \leq\left(\int_{0-}^{t}+\int_{T_{0}}^{\infty}\right) \bar{F}\left(x e^{r s}\right) d E N(s) \\
& \leq(1+\varepsilon) \int_{0-}^{t} \bar{F}\left(x e^{r s}\right) d E N(s) .
\end{aligned}
$$

On the other hand, by (3.14) with $t$ replaced by $T_{0}$ and (3.25), it holds uniformly for all $t \in\left(T_{0}, \infty\right]$ that

$$
\begin{aligned}
P\left(D_{r}(t)>x\right) & \geq P\left(D_{r}\left(T_{0}\right)>x\right) \sim \int_{0-}^{T_{0}} \bar{F}\left(x e^{r s}\right) d E N(s) \\
& \geq \frac{1}{1+\varepsilon} \int_{0-}^{\infty} \bar{F}\left(x e^{r s}\right) d E N(s) \\
& \geq \frac{1}{1+\varepsilon} \int_{0-}^{t} \bar{F}\left(x e^{r s}\right) d E N(s) .
\end{aligned}
$$


Consequently, by (3.27) and (3.28), it holds that, for all $t \in\left(T_{0}, \infty\right]$ and all large $x$,

$$
(1-2 \varepsilon) \int_{0-}^{t} \bar{F}\left(x e^{r s}\right) d E N(s) \leq P\left(D_{r}(t)>x\right) \leq(1+2 \varepsilon) \int_{0-}^{t} \bar{F}\left(x e^{r s}\right) d E N(s) .
$$

From the first part of this proof, we see that (3.29) still holds for all $t \in\left[t_{0}, T_{0}\right]$ and all large $x$. As a result, (3.29) holds for all $t \in\left[t_{0}, \infty\right]$ and all large $x$. Since $\varepsilon>0$ can be arbitrarily close to 0 , we conclude that relation (3.14) holds uniformly for all $t \in\left[t_{0}, \infty\right]$.

\section{Proof of main results}

We now proceed to prove Theorem 2.1. From the surplus process (1.1), we can get its discounted value as

$$
\widetilde{U}_{r}(t)=e^{-r t} U_{r}(t)=x+\widetilde{C}(t)-D_{r}(t), \quad t \geq 0,
$$

where $\widetilde{C}(t)=\int_{0-}^{t} e^{-r s} C(d s)$ and denotes the discounted value of premiums accumulated up to time $t>0$. Obviously, one can know by Assumption 3 on $\{C(t), t \geq 0\}$ that $0 \leq \widetilde{C}(t)<\infty$ almost surely for any fixed $t>0$. By the definition (1.2) of the finite-time ruin probability, we have

$$
\psi_{r}(x, t)=P\left(D_{r}(s)>x+\widetilde{C}(s) \text { for some } 0<s \leq t\right) .
$$

Then, it is easy to obtain that for any $t \in \Lambda$,

$$
\psi_{r}(x, t) \leq P\left(D_{r}(t)>x\right)
$$

$$
\psi_{r}(x, t)=P\left(\bigcup_{0<s \leq t}\left\{D_{r}(s)>x+\widetilde{C}(s)\right\}\right) \geq P\left(D_{r}(t)>x+\widetilde{C}(t)\right) .
$$

For the uniform asymptotic upper bound for $\psi_{r}(x, t)$ under conditions 1 and 2 , it follows immediately from (4.1) and Lemma 3.4 that, uniformly for all $t \in\left[t_{0}, \infty\right]$,

$$
\psi_{r}(x, t) \lesssim \int_{0-}^{t} \bar{F}\left(x e^{r s}\right) d E N(s) .
$$

Hence, it remains to derive the corresponding uniform asymptotic lower bound for $\psi_{r}(x, t)$, that is,

$$
\psi_{r}(x, t) \gtrsim \int_{0-}^{t} \bar{F}\left(x e^{r s}\right) d E N(s)
$$

holds uniformly for all $t \in\left[t_{0}, \infty\right]$. In the following, we formulate the proof of the uniformity of (4.3) into two parts.

In the first part, we deal with the case of condition 1 . Now we establish the local uniformity of relation (4.3) for all $t \in\left[t_{0}, T\right]$, where $T \in\left(t_{0}, \infty\right)$. By (4.2), 
Lemma 3.4, $F \in \mathcal{C} \subset \mathcal{L}$ and the local uniformity of (1.3), it holds uniformly for all $t \in\left[t_{0}, T\right]$ that

$$
\begin{aligned}
\psi(x, t) & \geq P\left(D_{r}(t)>x+\widetilde{C}(T)\right) \\
& =\int_{0-}^{\infty} P\left(D_{r}(t)>x+y\right) P(\widetilde{C}(T) \in d y) \\
& \sim \int_{0-}^{\infty} \int_{0-}^{t} \bar{F}\left((x+y) e^{r s}\right) d E N(s) P(\widetilde{C}(T) \in d y) \\
& \sim \int_{0-}^{\infty} \int_{0-}^{t} \bar{F}\left(x e^{r s}\right) d E N(s) P(\widetilde{C}(T) \in d y) \\
& =\int_{0-}^{t} \bar{F}\left(x e^{r s}\right) d E N(s) .
\end{aligned}
$$

Subsequently, we deal with the uniformity of $(4.3)$ for all $t \in(T, \infty]$ under condition 1. By (4.2), Lemma 3.4 with $t$ replaced by $T, F \in \mathcal{C} \subset \mathcal{L}$ and the local uniformity of (1.3), we prove that, uniformly for all $t \in(T, \infty]$,

$$
\begin{aligned}
\psi(x, t) & \geq P\left(D_{r}(T)>x+\widetilde{C}\right) \\
& =\int_{0-}^{\infty} P\left(D_{r}(T)>x+y\right) P(\widetilde{C} \in d y) \\
& \sim \int_{0-}^{\infty} \int_{0-}^{T} \bar{F}\left((x+y) e^{r s}\right) d E N(s) P(\widetilde{C} \in d y) \\
& \sim \int_{0-}^{\infty} \int_{0-}^{T} \bar{F}\left(x e^{r s}\right) d E N(s) P(\widetilde{C} \in d y) \\
& =\int_{0-}^{T} \bar{F}\left(x e^{r s}\right) d E N(s) \\
& \geq \frac{1}{1+\varepsilon} \int_{0-}^{t} \bar{F}\left(x e^{r s}\right) d E N(s)
\end{aligned}
$$

where in the last step we used (3.25). Because $\varepsilon>0$ is arbitrary, the relation (4.3) holds uniformly for all $t \in(T, \infty]$. So, we can obtain the uniformity of (4.3) over all $t \in\left(t_{0}, \infty\right]$ under condition 1 .

In the second part, we turn to the case of condition 2. First, we consider the local uniformity of relation (4.3) for all $t \in\left[t_{0}, T\right]$. Since $F \in \mathcal{C}$, we see that for any fixed $\varepsilon>0$, there exits some $\delta_{0}>0$ such that for all large $x$,

$$
\bar{F}\left(\left(1+\delta_{0}\right) x\right) \geq(1-\varepsilon) \bar{F}(x) .
$$

It follows from (4.2) that for $\delta_{0}>0$ as above and all $t \in\left[t_{0}, T\right]$,

$$
\begin{aligned}
\psi_{r}(x, t) & \geq P\left(D_{r}(t)>x+\widetilde{C}(T)\right) \\
& \geq P\left(D_{r}(t)>\left(1+\delta_{0}\right) x\right)-P\left(\widetilde{C}(T)>\delta_{0} x\right)
\end{aligned}
$$




$$
=H_{1}-H_{2} .
$$

For $H_{1}$, by Lemma 3.4 and (4.4), it holds uniformly for all $t \in\left[t_{0}, T\right]$ that

$$
\begin{aligned}
H_{1} & \sim \int_{0-}^{t} \bar{F}\left(\left(1+\delta_{0}\right) x e^{r s}\right) d E N(s) \\
& \geq(1-\varepsilon) \int_{0-}^{t} \bar{F}\left(x e^{r s}\right) d E N(s) .
\end{aligned}
$$

For $H_{2}$, by condition 2 and $F \in \mathcal{C} \subset \mathcal{D}$, we get

$$
\limsup _{x \rightarrow \infty} \frac{H_{2}}{\bar{F}(x)}=\limsup _{x \rightarrow \infty} \frac{P\left(\widetilde{C}(T)>\delta_{0} x\right)}{P\left(\widetilde{C}>\delta_{0} x\right)} \cdot \frac{P\left(\widetilde{C}>\delta_{0} x\right)}{\bar{F}\left(\delta_{0} x\right)} \cdot \frac{\bar{F}\left(\delta_{0} x\right)}{\bar{F}(x)}=0 .
$$

This, along with (3.20), yields that for all $t \in\left[t_{0}, T\right]$,

$$
H_{2} \leq \varepsilon \bar{F}(x) \leq C_{0} \varepsilon \int_{0-}^{t_{0}} \bar{F}\left(x e^{r s}\right) d E N(s) \leq C_{0} \varepsilon \int_{0-}^{t} \bar{F}\left(x e^{r s}\right) d E N(s) .
$$

Hence, substituting (4.6) and (4.7) into (4.5) and using the arbitrariness of $\varepsilon>0$, we derive that relation (4.3) holds uniformly for all $t \in\left[t_{0}, T\right]$.

Next, we focus on the uniformity of (4.3) for all $t \in(T, \infty]$ under condition

2. Let $\delta_{0}>0$ be fixed as above, by (4.2) we find that

$$
\begin{aligned}
\psi_{r}(x, t) & \geq P\left(D_{r}(T)>x+\widetilde{C}\right) \\
& \geq P\left(D_{r}(T)>\left(1+\delta_{0}\right) x\right)-P\left(\widetilde{C}>\delta_{0} x\right) \\
& =H_{3}-H_{4} .
\end{aligned}
$$

For $H_{3}$, by Lemma 3.4 with $t$ replaced by $T$, (4.4) and (3.25), it holds uniformly for all $t \in(T, \infty]$ that

$$
\begin{aligned}
H_{3} & \sim \int_{0-}^{T} \bar{F}\left(\left(1+\delta_{0}\right) x e^{r s}\right) d E N(s) \\
& \geq(1-\varepsilon) \int_{0-}^{T} \bar{F}\left(x e^{r s}\right) d E N(s) \\
& \geq(1-2 \varepsilon) \int_{0-}^{t} \bar{F}\left(x e^{r s}\right) d E N(s) .
\end{aligned}
$$

For $H_{4}$, by condition 2 and $F \in \mathcal{C} \subset \mathcal{D}$, we get that for all $t \in(T, \infty]$,

$$
\begin{aligned}
\limsup _{x \rightarrow \infty} \frac{H_{4}}{\int_{0}^{t} \bar{F}\left(x e^{r s}\right) d E N(s)} & \leq \limsup _{x \rightarrow \infty} \frac{H_{4}}{\int_{0-}^{T} \bar{F}\left(x e^{r s}\right) d E N(s)} \\
& \leq \limsup _{x \rightarrow \infty} \frac{H_{4}}{\bar{F}\left(\delta_{0} x\right)} \cdot \frac{\bar{F}\left(\delta_{0} x\right)}{\bar{F}\left(x e^{r T}\right) E N(T)} \\
& =0,
\end{aligned}
$$


which implies that for all $t \in(T, \infty]$ and all large $x$,

$$
H_{4} \leq \varepsilon \int_{0-}^{t} \bar{F}\left(x e^{r s}\right) d E N(s) .
$$

Consequently, by (4.8)-(4.10) and the arbitrariness of $\varepsilon>0$, we can obtain the uniformity of (4.3) for all $t \in(T, \infty]$, and then relation (4.3) holds uniformly for all $t \in\left[t_{0}, \infty\right]$ under condition 2 .

Acknowledgement. The authors thank the editors and an anonymous referee for their helpful comments on the paper.

\section{References}

[1] N. H. Bingham, C. M. Goldie, and J. L. Teugels, Regular Variation, Cambridge University Press, Cambridge, 1987.

[2] Y. Chen and K. Yuen, Sums of pairwise quasi-asymptotically independent random variables with consistent variation, Stoch. Models 25 (2009), no. 1, 76-89.

[3] P. Embrechts, C. Kluuppelberg, and T. Mikosch, Modelling Extremal Events, For Insurance and Finance, Springer, Berlin, 1997.

[4] X. Hao and Q. Tang, A uniform asymptotic estimate for discounted aggregate claims with subexponential tails, Insurance Math. Econom. 43 (2008), no. 1, 116-120.

[5] H. Jasiulewicz, Probability of ruin with variable premium rate in a Markovian environment, Insurance Math. Econom. 29 (2001), no. 2, 291-296.

[6] F. Kong and G. Zong, The finite-time ruin probability for ND claims with constant interest force, Statist. Probab. Lett. 78 (2008), no. 17, 3103-3109.

[7] S. Kotz, N. Balakrishnan, and N. L. Johnson, Continuous Multivariate Distributions. Vol. 1, Models and applications. Second edition. Wiley Series in Probability and Statistics: Applied Probability and Statistics. Wiley-Interscience, New York, 2000.

[8] F. Michaud, Estimating the probability of ruin for variable premiums by simulation, Astin Bull. 26 (1996), no. 1, 93-105.

[9] S. S. Petersen, Calculation of ruin probabilities when the premium depends on the current reserve, Scand. Actuar. J. 1898 (1989), no. 3, 147-159.

[10] Q. Tang and G. Tsitsiashvili, Precise estimates for the ruin probability in finite horizon in a discrete-time model with heavy-tailed insurance and financial risks, Stochastic Process. Appl. 108 (2003), no. 2, 299-325.

[11] _ Randomly weighted sums of subexponential random variables with application to ruin theory, Extremes 6 (2003), no. 3, 171-188.

[12] Q. Tang, Asymptotic ruin probabilities of the renewal model with constant interest force and regular variation, Scand. Actuar. J. 2005 (2005), no. 1, 1-5.

[13] - Heavy tails of discounted aggregate claims in the continous-time renewal model, J. Appl. Probab. 44 (2007), no. 2, 285-294.

[14] D. Wang, Finite-time ruin probability with heavy-tailed claims and constant interest rate, Stoch. Models 24 (2008), no. 1, 41-57.

[15] K. Wang, Y. Wang, and Q. Gao, Uniform asymptotics for the finite-time ruin probability of a dependent risk model with a constant interest rate, Methodol. Comput. Appl. Probab. 15 (2013), no. 1, 109-124.

[16] Y. Yang and Y. Wang, Asymptotics for ruin probability of some negatively dependent risk models with a constant interest rate and dominatedly-varying-tailed claims, Statist. Probab. Lett. 80 (2010), no. 3-4, 143-154.

[17] L. Yi, Y. Chen, and C. Su, Approximation of the tail probability of randomly weighted sums of dependent random variables with dominated variation, J. Math. Anal. Appl. 376 (2011), no. 1, 365-372. 
QINGWU GaO

School of Mathematics and Statistics

Nanjing Audit University

NAnjing, 211815, P. R. China

E-mail address: qwgao@yahoo.cn

YANG YANG

School of Mathematics and Statistics

NAnjing Audit University

NAnjing, 211815, P. R. ChinA

E-mail address: yyang@nau.edu.cn 